\title{
NEUROPATÍA EN EL CONTEXTO DEL SÍNDROME METABÓLICO Y DE LA DISGLUCEMIA NO DIABÉTICA
}

\author{
NEUROPATHY IN METABOLIC SYNDROME \\ AND NON-DIABETIC DYSGLYCEMIA
}

Comité de Neuropatía Diabética de la Sociedad Argentina de Diabetes: Carolina Domínguez', Claudia Flores $^{2}$, Graciela Fuente ${ }^{1}$, Concepción García ${ }^{1}$, Marcela Giménez Rey ${ }^{3}$, Solange Houssay ${ }^{4}$, Fernanda Huber ${ }^{5}$, Carlos Santillán' ${ }^{6}$ Raquel Urdaneta ${ }^{1}$

\section{RESUMEN}

La neuropatía diabética (ND) es la complicación microvascular más frecuente y más precoz asociada a la diabetes mellitus (DM), no obstante esta situación, es la más olvidada y la menos diagnosticada. Al mismo tiempo es de amplio conocimiento que el síndrome metabólico (SM) tiene una elevada prevalencia, y que la relación entre este estado y el compromiso macrovascular está documentada. Es de interés para el Comité de Neuropatía Diabética analizar la afectación neuropática en el marco del SM y en el de uno de sus componentes constitutivos como es la disglucemia no diabética. El subdiagnóstico de esta entidad y el aumento del riesgo de la morbimortalidad de los pacientes que la padecen hacen importante alertar a la comunidad médica de la existencia de esta complicación microvascular que excede el marco de la DM y se presenta también en el SM y en la disglucemia tanto de ayunas como posprandial.

Palabras clave: neuropatía diabética, síndrome metabólico, disglucemia no diabética.

Revista de la Sociedad Argentina de Diabetes 2015; Vol. 49 (69-74)

\section{ABSTRACT}

Diabetic neuropathy (DN) is the most common and early microvascular alteration associated to diabetes mellitus (DM); however, this event is most missed and less diagnosed. At the same time, it is widely known that metabolic syndrome (MS) is highly prevalent and the relationship between this status and macrovascular commitment is documented. The Diabetic Neuropathy Committee is focused in analyzing the neuropathic effect within the framework of MS and in one of its components such as non-diabetic disglycemia. The under diagnosis and increase of morbidity and mortality risk of these patients alert the medical community of the existence of this microvascular alteration exceeding the DM framework and is also present in MS and disglycemia either at fasting and postprandial.

Key words: diabetic neuropathy, metabolic syndrome, nondiabetic disglycemia.

Revista de la Sociedad Argentina de Diabetes 2015; Vol. 49 (69-74)

\section{Hospital Durand, CABA, Argentina}

Hospital Penna, Bahía Blanca, Prov. de Buenos Aires, Argentina

3 Hospital Domingo Mercante, Prov. de Buenos Aires, Argentina

Hospital Tornú y Clínica San Camilo, CABA, Argentina

5 Comité de Neuropatía de la SAD, CABA, Argentina

6 Hospital Dr. Leónidas Lucero, Bahía Blanca, Prov. de Buenos Aires, Argentina

Contacto del autor: Raquel Urdaneta

E-mail: raquelurdaneta@live.com.ar

Correspondencia: Servicio de Nutrición y Metabolismo. Hospital General de Agudos

Dr. E. Tornú. Combatientes de Malvinas 3002 (C1427ARN), CABA, Argentina

Fecha de trabajo recibido: 2/6/15

Fecha de trabajo aceptado: 27/6/15 


\section{INTRODUCCIÓN}

La diabetes tipo 2 ha alcanzado proporciones epidémicas. La polineuropatía distal y simétrica y la neuropatía autonómica son trastornos evolutivos de la diabetes asociados con mayor morbilidad y mortalidad. El concepto tradicional consideraba que la neuropatía diabética se presentaba luego de largo tiempo de evolución de la enfermedad de base, pero recientes evidencias indican que puede estar presente en etapas más tempranas, aún antes del diagnóstico de la diabetes.

Los valores alterados de la glucosa se han visto con mayor frecuencia implicados en la etiopatogenia de las neuropatías de causa idiopática. Además, otras anormalidades metabólicas como la obesidad abdominal, hipertensión arterial, hipertrigliceridemia sola o asociada a HDL bajo configuran el denominado síndrome metabólico que puede asociarse con neuropatía?.

\section{Definiciones}

- Disglucemia. Se incluye en este término glucemia alterada en ayunas (GAA): glucemia en ayunas entre 110 y $125 \mathrm{mg} / \mathrm{dl}$ y tolerancia alterada a la glucosa (TAG): glucemia a las 2 horas en la prueba de tolerancia oral a la glucosa (PTOG) entre 140 y $199 \mathrm{mg} / \mathrm{dl}$.

- Síndrome metabólico (SM). Se denomina SM al conjunto de alteraciones metabólicas constituido por la obesidad de distribución central, la diminución de las concentraciones del colesterol unido a las lipoproteínas de alta densidad (HDL), la elevación de las concentraciones de triglicéridos, el aumento de la presión arterial y la hipergluce$\mathrm{mia}^{2}$. Existen varias clasificaciones. Utilizaremos la clasificación según el Adult Treatment Panel III (NCEP-ATP III) ${ }^{3}$ que define SM con tres o más componentes de cinco. Tiene en cuenta: perímetro de cintura (hombre mayor a $102 \mathrm{~cm}$, mujer a 88 $\mathrm{cm}$ ), hipertensión arterial (mayor a 130/85 mmHg), hipertrigliceridemia (mayor a $150 \mathrm{mg} / \mathrm{dl}$ ), colesterol HDL (hombre menor de $40 \mathrm{mg} / \mathrm{dl}$ y mujer menor de $50 \mathrm{mg} / \mathrm{dll}$ ), glucemia en ayunas (glucemia $\geq 110 \mathrm{mg} / \mathrm{dl}$ ).

- Neuropatía idiopática. La polineuropatía crónica axonal idiopática (PCAI o CIAP por sus siglas en inglés) es una neuropatía predominantemente sensorial o sensitivo-motora que aparece en la sexta década de la vida, progresa muy lentamente y no produce discapacidad. Anatomopatológicamente tiene una similitud con la polineuropatía diabética, lo que sugiere un mecanismo fisiopatológico similar ${ }^{4}$.

\section{Epidemiología}

La relación entre disglucemia y neuropatía idiopática es bidireccional dado que una proporción sustancial de individuos con neuropatía idiopática tiene disglucemia (25 a $62 \%$ ) y una proporción importante de pacientes con disglucemia presenta neuropatía (11 a 25\%) o dolor neuropático (13 a 21\%).

Existen distintos estudios que proveen evidencia de la asociación entre componentes del síndrome metabólico excluyendo la disglucemia y la neuropatía periferica ${ }^{5}$.

El reconocimiento de una relación potencial entre disglucemia y neuropatía ha tenido origen en la observación clínica de muchos pacientes que comparten características fenotípicas de diabetes tipo 2 como obesidad, hipertensión y dislipidemia sin tener diabetes diagnosticada6.

El SM provee un elevado riesgo de neuropatía, aún en ausencia de incremento de la glucosa. En un estudio que incluyó a 219 pacientes con neuropatía periférica idiopática se determinó la prevalencia de los componentes del SM; se clasificó a los pacientes según tuvieran normoglucemia o intolerancia a la glucosa. Ambos grupos compartieron una prevalencia similar de los distintos componentes del SM, si bien el grupo de normoglucémicos con neuropatía presentó mayores niveles de LDL y TG con valores más bajos de HDL4. Consistentemente con esto, en la literatura se sugiere que el SM, particularmente la dislipidemia y la obesidad, son potentes factores de riesgo para el desarrollo tanto de neuropatía diabética como idiopática ${ }^{6,7}$.

En el estudio MONICA/KORA (Monitorig trends and determinants in Cardiovascular Cooperative Research in the Region of Augsburg), se informa una prevalencia de neuropatía del $28 \%$ de los sujetos diabéticos, el $13 \%$ en TAG, el $11,3 \%$ en GAA y el $7,4 \%$ en normoglucémicos. El dolor neuropático fue también más frecuente en los sujetos con diabetes observándose en el 13,3\% de los mismos, el $8,7 \%$ en TAG, el $4,2 \%$ en GAA y el $1,2 \%$ en normoglucémicos ${ }^{7}$. En el mismo estudio se evaluaron sobrevivientes de infarto de miocardio con dolor neuropático, los que presentaron diabetes el $21 \%$, TAG el $14,8 \%$, GAA el $5,7 \%$ y el $3,7 \%$ normoglucemia. Estos datos proveen una fuerte evidencia que en la población general el dolor neuropático es aproximadamente dos a cuatro veces más frecuen- 
te en sujetos con diabetes e intolerancia que en aquellos con GAA y normoglucemia ${ }^{9,10}$.

\section{Fisiopatología}

El compromiso neuropático se presenta en estado de hiperglucemia, aún en un período incipiente de la misma y en estados de dismetabolismo como ocurre cuando están presentes los componentes del SM sin hiperglucemia manifiesta.

La vía etiológica cardinal para el desarrollo de neuropatía en personas con TAG es la hiperglucemia. En una publicación reciente del estudio poblacional KORA, el tercer y cuarto cuartilo de glucosa a las dos horas post carga fueron factores de riesgo independiente de neuropatía. En la vida real los pacientes con TAG desarrollan hiperglucemia intermitente principalmente en el período postprandial y esto se repite constantemente ${ }^{8}$. Existen amplias evidencias experimentales y clínicas de que la hiperglucemia aguda transitoria genera estrés oxitadativo y disfunción endotelial produciendo alteración de la función mitocondrial, metabolismo celular alterado y daño del ADN a nivel neuronal. En perros se demostró que la hiperglucemia postprandial reduce la dilatación mediada por el flujo y aumenta la concentración de metilglioxal. Este último es un intermediario de la glucosa dicarbonílico altamente reactivo inductor de estrés oxidativo. En ratas el metilglioxal se asocia con reducción de la velocidad de conducción nerviosa, despolarización de las neuronas sensitivas e hiperalgesia mecánica y térmica. Cuando la hiperglucemia se hace crónica sus efectos deletéreos son mediados por la activación de la PKC y la vía de los polioles que conlleva un mayor daño neuronal ${ }^{8}$.

Un segundo mecanismo muy importante es la dislipidemia. Hay amplias evidencias experimentales que sugieren que la misma tiene un efecto neurotóxico sustancial que se suma al producido por las excursiones glucémicas. Los triglicéridos elevados constituyen el principal factor de riesgo para la neuropatía axonal crónica idiopática ${ }^{11}$.

Existe evidencia en modelos animales que la dieta rica en grasas produce aumento de ácidos grasos libres (AGL) e hiperinsulinemia que se traduce en déficit motor y sensitivo en estudios de conducción nerviosa. En ratas se presenta alodinia e hipoalgesia térmica sin pérdida de fibras nerviosas intraepidérmicas o atrofia axonal. Entre los mecanismos involucrados se describe el aumento de la vía del sorbitol, de 4-hidroxi-2-nonenal nitroti- rosina, de poli-ADP-ribosa y de 12,15 lipooxigenasa, que se sobreexpresan en nervios periféricos y ganglios anexos a la raíz dorsal; estas alteraciones se corrigen con dieta normal e inhibidores de la aldosa reductasa. Este modelo animal desarrolla alteraciones funcionales y no estructurales con la dieta alta en grasa y constituye un modelo para evaluar intervenciones dietarias y farmacológicas para detener y revertir la neuropatía diabética en estadios precoces ${ }^{12}$.

Diversos estudios, empleando modelos animales, muestran disminución de la relajación vascular y alteración de la velocidad de conducción nerviosa, con disminución del flujo sanguíneo endoneural y de la sensibilidad térmica ${ }^{13,14,15}$. Ratones no diabéticos alimentados con dietas ricas en grasas desarrollaron incrementos en los niveles de LDL oxidados, AGL y TG así como también evidenciaron incremento en el estrés oxidativo sistémico y a nivel del nervio. Estos ratones desarrollaron déficits en la velocidad de conducción nerviosa y en la sensibilidad antes de desarrollar TAG ${ }^{16}$. Estos hallazgos son altamente relevantes para entender la neuropatía observada en humanos con insulinorresistencia, obesidad y dislipidemia ${ }^{17}$.

Las personas con neuropatía idiopática tienen mayor número de características del síndrome metabólico diferentes de la hiperglucemia (predominantemente obesidad y dislipidemia) que aquellas sin neuropatía, implicándolo per se en el desarrollo de la neuropatía. La obesidad abdominal también surgió como factor de riesgo para neuropatía en personas de la población general en el estudio KORA. El rol de la obesidad es mediado a través de su asociación con la dislipidemia y la mayor producción de citoquinas inflamatorias. Las citoquinas inflamatorias producen un efecto neurotóxico dual directa e indirectamente a través de un aumento del estrés oxidativo ${ }^{11}$.

La obesidad puede conducir al aumento de TNF-alfa y lípidos circulantes (AGL y TG) que, por un lado, pueden aumentar la glucemia promoviendo la gluconeogénesis hepática, y por el otro actúan independientemente sobre la función del nervio a través del incremento del estrés oxidativo y la disfunción endotelial ${ }^{18}$. Cada vez más evidencia sugiere que el incremento del estrés oxidativo, particularmente el aumento del peroxinitrito, causa daño sobre distintos tejidos incluyendo el nervio periférico ${ }^{19,20,21,22,23,24}$.

La neuropatía de fibra fina es un desorden de 
los nervios periféricos que afecta primaria o exclusivamente las pequeñas fibras somáticas y/o autonómicas resultando en alteraciones sensoriales y disfunción autonómica. Las pequeñas fibras incluyen las fibras mielinizadas A delta y las no mielinizadas $\mathrm{C}$ que inervan la piel (fibras somáticas) y los músculos involuntarios, incluyendo el cardíaco y el liso (fibras autonómicas). Juntas median el dolor, la sensación térmica y la función autonómica; la neuropatía de fibras finas resulta del compromiso selectivo de las pequeñas fibras mielinizadas A delta y no mielinizadas $\mathrm{C}^{25,26}$.

\section{Clínica}

La neuropatía se manifiesta clínicamente por síntomas sensitivos y autonómicos. Los síntomas provocados por el daño o la pérdida de fibras somáticas finas son dolor, ardor, parestesias o adormecimiento que afectan típicamente los miembros inferiores con un gradiente distal a proximal.

El síntoma más molesto es el dolor que se extiende en forma proximal (en media), que puede ser urente, punzante, lacerante o presentarse en forma de calambres u hormigueos. En ocasiones pueden comprometerse los miembros superiores en forma de guante. Los síntomas suelen empeorar de noche y alteran la calidad del sueño.

El examen clínico revela alodinia (percepción de un estímulo no doloroso como doloroso), hiperalgesia (percepción de un estímulo doloroso como más doloroso de lo esperado) o disminución de la sensibilidad discriminativa (prueba toco-pincho) y térmica en el área afectada. La sensibilidad vibratoria puede estar levemente reducida en los hallux. La fuerza motora, los reflejos osteotendinosos y la propiocepción están preservados porque son funciones de fibras largas.

Cuando se afectan las fibras autonómicas los pacientes experimentan ojo seco, boca seca, intolerancia ortostática, taquicardia de reposo, hipotensión ortostática sin taquicardia compensadora, constipación, incontinencia vesical, disfunción sexual, síndrome de anhidrosis con cambios en la piel (atrófica, seca, edematosa, decolorada, brillosa) por anormalidades sudomotoras y vasomotoras.

La neuropatía de fibra fina se confunde con frecuencia con la fascitis plantar, insuficiencia vascular o enfermedad vertebral degenerativa lumbosacra ${ }^{26}$.

\section{Diagnóstico y diagnósticos diferenciales}

Ante la sospecha clínica de neuropatía de fibra fina se debe realizar un examen neurológico exhaustivo para descartar otras neuropatías.

Se recomienda realizar una valoración del metabolismo glucídico como parte del algoritmo para realizar diagnóstico de neuropatía idiopática ${ }^{27}$.

Ante dudas diagnósticas se utilizan estudios de velocidad de conducción nerviosa y electromiográficos. Estos test deberían ordenarse para excluir el compromiso de fibras largas que podrían afectar el diagnóstico, el pronóstico y el plan de tratamiento. Si estos resultados son normales serán necesarios estudios especializados para evaluar el compromiso de fibra fina ${ }^{26}$.

El diagnóstico de certeza se realiza con biopsia de piel y los test cuantitativos de reflejo sudomotor axónico - OSART (Quantitative Sudomotor Axon Reflex Test) y la prueba sensitiva cuantitativa -QST (Quantitative Sensitive Test). Los test se emplean para valorar la función autonómica sudomotora y determinar el estado funcional evolutivo de las sensibilidades vibratorias, térmicas y termoalgésicas. EI QST tiene una sensibilidad y reproducibilidad superior al $80 \%$.

Con la biopsia se evalúa la densidad de las fibras nerviosas intraepidérmicas, que suele estar disminuida o normal en las primeras etapas pero muestra cambios morfológicos, especialmente edema importante. La biopsia de piel tiene una eficacia diagnóstica del $88 \%$, es más sensible y menos invasiva que la biopsia del nervio sural.

Se han identificado antes del desarrollo de neuropatía en TAG anormalidades capilares en biopsia de nervio sural y en la piel con una menor vasodilatación dependiente del endotelio inducida por acetilcolina, lo que estaría implicado en su patogenia ${ }^{28}$.

Si estos test no están disponibles, es importante realizar una batería de análisis de laboratorio básicos para hallar la causa subyacente: hemograma, eritrosedimentación, perfil lipídico, perfil tiroideo, perfil reumatológico, vitamina B12, serología para virus de la inmunodeficiencia humana $(\mathrm{VIH})$, perfil metabólico, anticuerpos para descartar celiaquía y la prueba de tolerancia a la glucosa (PTOG); se debe solicitar en todos los pacientes con síntomas de neuropatía de fibra fina sin historia conocida de diabetes o prediabetes. Otros estudios específicos se requerirán de acuerdo a la presunción clínica ${ }^{22,23}$.

La neuropatía de fibra fina se ha asociado a 
distintas condiciones médicas incluyendo dismetabolismo de glucosa, enfermedades del tejido conectivo, enfermedades tiroideas, deficiencia de vitamina B12, paraproteinemias, VIH, infecciones por virus de la hepatitis $\mathrm{C}(\mathrm{HCV})$, síndrome de piernas inquietas, exposición a drogas neurotóxicas y enfermedades hereditarias; estas patologías producen neuropatía de fibra fina dependiente de la longitud ${ }^{25}$ mientras que síndromes paraneoplásicos, la enfermedad celíaca y la enfermedad de Sjögren son de pequeña fibra sin depender de la longitud de la misma $29,30,31,32$.

\section{Tratamiento}

El tratamiento de la neuropatía de fibra fina debe focalizarse en la causa subyacente y en la sintomatología dolorosa.

Como la tolerancia alterada a la glucosa es la condición más frecuentemente asociada con la neuropatía de fibra fina y como los componentes individuales del síndrome metabólico son factores de riesgo potenciales, el cambio terapéutico del estilo de vida con reducción del peso corporal, la actividad física regular, la corrección de factores de riesgo cardiovasculares y el control glucémico, en caso que se requiera, son fundamentales en esta condición.

El Diabetes Prevention Programe (DPP) ${ }^{33}$, un estudio de 3.224 pacientes con prediabetes, publicó que la dieta y el ejercicio son más efectivos que la metformina en prevenir la diabetes luego de 2,8 años de seguimiento. El riesgo de diabetes se redujo en un $31 \%$ en el grupo tratado con metformina en comparación con una reducción del 58\% en los pacientes asignados a cambio terapéutico en el estilo de vida. El cambio terapéutico de estilo de vida incluyó 150 minutos de actividad física semanal y reducción de peso de un 7\% del peso inicial. Dicho de otra manera, para prevenir un caso de diabetes en tres años 6,9 pacientes deben ser incluidos en un programa de intervención de estilo de vida o 13,9 deben recibir metformina. Como la neuropatía de la TAG representa la etapa más temprana de la neuropatía diabética, la neuropatía en este estadio podría ser reversible con intervención en el estilo de vida y mejoría en la tolerancia a la glucosa ${ }^{34}$. Este concepto se basa en un estudio a tres años en 31 personas que demostró que un programa de cambio de estilo de vida mejoró significativamente la TAG y redujo el índice de masa corporal (IMC) y el colesterol total ${ }^{35}$.
Cambios en estas variables metabólicas se acompañaron de mejoría significativa de la neuropatía medida como un aumento en la densidad de las fibras nerviosas intraepidérmicas, aumento en el volumen de sudoración del pie y reducción del dolor neuropático.

Cabe mencionar que en caso de neuropatía causada por otras enfermedades como la sarcoidosis, patologías autoinmunes y enfermedad celíaca los síntomas mejoran con su tratamiento específico.

\section{Tratamiento sintomático}

El manejo del dolor es crucial en el tratamiento de NFF y como puede ser debilitante y causante de depresión, requiere de un manejo multidisciplinario. Las drogas disponibles en nuestro medio para el tratamiento de NFF se detallan en la Tabla 1.

\begin{tabular}{|ccc|}
\hline Droga & Dosis diaria & Efectos adversos comunes \\
\hline ANTIDEPRESIVOS & \\
\hline Amitriptilina & $20-150 \mathrm{mg}$ & $\begin{array}{l}\text { Sedación, ganancia de peso, } \\
\text { efectos }\end{array}$ \\
\hline Duloxetina & $60-120 \mathrm{mg}$ & anticolinérgicos, arritmia \\
\hline
\end{tabular}

\begin{tabular}{|lll|}
\hline ANTICONVULSIONANTES & \\
\hline Gabapentin & $\begin{array}{l}600-3600 \\
\mathrm{mg}\end{array}$ & $\begin{array}{l}\text { Sedación, mareos, } \\
\text { edema periférico, }\end{array}$ \\
\hline Pregabalina & $150-600 \mathrm{mg}$ & ganancia de peso \\
\hline
\end{tabular}

\section{OPIOIDES, OPIOIDES AGONISTAS}
Tramadol 100-400 mg Sedación, mareos, nausea, constipación,

Oxicodona $\quad 10-100 \mathrm{mg}$ potencial adicción y abuso

Tabla 1: Drogas utilizadas para control del dolor en neuropatía de fibra fina ${ }^{25}$.

\section{CONCLUSIONES}

En la actualidad existen evidencias de la asociación de neuropatía con tolerancia alterada a la glucosa y en menor grado con glucemia alterada en ayunas. La relación es bidireccional, existe una proporción de pacientes con neuropatía idiopática que 
tiene disglucemia y sujetos con disglucemia que presentan neuropatía periférica o dolor neuropático.

Si bien el estudio MONICA/KORA fue realizado con un número importante de participantes, la mayoría de los estudios tiene un tamaño muestral pequeño y fue realizado en pacientes caucásicos. Es necesario, por lo tanto, contar con estudios epidemiológicos que se focalicen en el análisis de pacientes con síndrome metabólico con o sin diabetes, utilizando estudios de diseño longitudinal y empleando rigurosas definiciones de neuropatía. Esta información podría brindar evidencia de las relaciones entre el síndrome metabólico y la neuropatía. También se debería definir el impacto de cada uno de los componentes del síndrome metabólico y su rol en el desarrollo de la neuropatía.

\section{REFERENCIAS}

1. Ylitalo KR, Sowers M, Heeringa S. Peripheral vascular disease and peripheral neuropathy in individuals with cardiometabolic clustering and obesity. National Health and Nutrition Examination Survey 2001-2004. Diabetes Care 2011; 34:1642-1647.

2. Eckel EH, Grundy SM, Zimmet PZ. The metabolic syndrome. Lancet 2005; 365: 1415-28.

3. Expert Panel on Detection, Evaluation and Treatment of High Blood Cholesterol in Adults. Executive Summary of The Third Report of The National Cholesterol Education Program (NCEP) Expert Panel on Detection, Evaluation, and Treatment of High Blood Cholesterol In Adults (Adult Treatment Panel III). JAMA 2001; 285:2486-97.

4. Gordon Smith A, Kristi Rose J, Singleton R. Idiopathic neuropathy patients are at high risk for metabolic syndrome. J. Neurol. Sci. 2008; October 15; 273(1-2): 25-28.

5. Bongaerts BWC, Rathmann W, Heier M, et al. Older subjects with diabetes and prediabetes are frequently unaware of having distal sensorimotor polyneuropathy. The KORA F4 Study. Diabetes Care 2013; 36: 1141-1146.

6. Gordon Smith A. Impaired glucose tolerance and metabolic syndrome in idiopathic neuropathy REVIEW. Journal of the Peripheral Nervous System 2012; 17 (Supplement):15-21.

7. Fuente G, Urdaneta Velez R, Lifszyc P. Neuropatía autonómica cardíaca en pacientes con síndrome metabólico. Pren. Med. Argent. 2011; 98: 469-475.

8. Vinik Al, Nevoret ML. Neuropathy in prediabetes and metabolic. In syndrome prevention of type 2 diabetes; from science to therapies (Cap. 8). Editor Derek LeRoith Department of Medicine Mount Sinai School of Medicine NewYork, USA. Springer Science Business Media, New York, 2012.

9. Bongaerts BWC, Rathmann W, Kowall B, et al. Postchallenge hyperglycemia is positively associated with diabetic polyneuropathy. The KORA F4 Study. Diabetes Care 2012; 35: 1891-1893.

10. Ziegler D, Rathman W, DickhausT, et al. Neuropathic pain in diabetes and normal glucose tolerance. The MONICA/KORA Augsburg Surveys S2 and S3. Pain Medicine 2009; 10 (2):393-400.

11. Hughes RA. A controlled investigation of the cause o chronical idiopathic axonal plyneuropathy. Brain 2004; 127; 1723-1730.

12. Obrosova IG, Ilnytska O, Lyzogubov VV, et al. High fat diet induced neuropathy of pre-diabetes and obesity. Effects of "healthy" diet and aldose reductase inhibition. Diabetes 2007; 56: 2598 - 2608.
13. Davinson EP, Coppey L, Calcut NA, et al. Diet-induced obesity in Sprague-Dawlwy rats causes microcascular and neural dysfunction. Diabetes Metab. Res. Rew. 2010; 26: 306-318.

14. Obrosova IG, Linytska O, LyzogubovW, et al. High fat diet induced neuropathy of pre-diabetes and obesity: effects of "healthy" diet and aldose reductase inhibition. Diabetes 2007; 56: 2598-2608.

15. Oltman CL, Coppey LJ, Gellett JS, et al. Progression of vascular and neural dysfunction in sciatic nerves of Zucker diabetic fatty and Zucker rats. Am. J. Phisiol. Endocrinol. Metab. 2005; 289: E113-E122.

16. Vincent AM, Hinder LM, Pop-Busui R, et al. Hyperlipemia: a new therapeutic target for diabetic neuropathy. J. Peripher Nerv. Syst. 2009; 14: 257-267.

17. Oltman CL, Davinson EP, Coppey LJ, et al. Treatment of Zucker diabetic fatty rats with AVE7688 improves vascular and neural dysfunction. Diabetes Obes. Metab. 2009; 11: 223-233.

18. Pleiner J, Schaler G, Mittermayer F, et al. FFA-induced endothelial dysfunction can be corrected by vitamin C. J. Clin. Endocrinol. Metab. 2002; 87: 2913-2917.

19. Smith AG, Singleton JR. Idiopatic neuropathy, prediabetes and metabolic syndrome (Abstract). J. Neuro. Sci 2006; 242: 9-14.

20. Testafaye $S$, Chaturvedi N, Eaton SE, et al. Vascular risk factors and diabetic neuropathy. N. Engl. J. Med. 2005; 352: 341-50.

21. Vincent AM, Hayes JM, McLean LL, et al. Dyslipidemia-induced neuropathy in mice: the rol of oxLDL/LOX-1. Diabetes 2009; 58: 2376-85.

22. Smith AG, Rose K, Singleton JR. Idiopathic neuropathy patients are high risk syndrome. J. Neurol. Sci. 2008; 273: 25-8.

23. Singleton JR, Smith AG, Russell JW, et al. Microvascular complications of impaired glucose tolerance. Diabetes 2003; 52: 2867-73.

24. Callaghan B, Feldman E. The Metabolic syndrome and neuropathy: therapeutic challenges and opportunities. Ann. Neurol. 2013; 74: 397-403.

25. Sumner CJ, Sheth S, Griffin JW, et al. The spectrum of neuropathy in diabetes and impaired glucose tolerance. Neurology 2003; 60; 108-111.

26. Tavee J, Zhou L. Small fiber neurophaty: a burning problem. Cleveland Clinic Journal of Medicine 2009; 76: 5; 297-305.

27. Papanas N, Vinik Al, Ziegler D. Neuropathy in prediabetes: does the clock start ticking early? Nat. Rev. Endocrinol. 2011; 7: $11 ; 682-690$.

28. Papanas N, Ziegler D. Polyneuropathy in impaired glucose tolerance: is postprandial hyperglucemia te main culprit? A minireview. Gerontology 2013; 59: 193-198.

29. McArthur JC, Brew BJ, Nath A. Neurological complications of HIV infection. Lancet Neurol. 2005; 4: 543-555.

30. Chain J, Herrmann DN, Staton M, et al. Pain ful samall- fiber neuropathy in Sjogren syndrome. Neurology 2005; 65: 925-927.

31. Brannagan $\mathrm{TH}, \mathrm{Hays} \mathrm{AP}$, Chin SS, et al. Small-fiber neuropathy/ neuropathy associated with celiac disease: skin biopsy findings. Arch. Neurol. 2005; 62: 1574-1578.

32. Gorson KC, Hermann DN, Thiagarajan R, et al. Nom-length dependent small fibre neuropathy/ganglionopathy. J. Neurol. Neurosurg. Psychiatric. 2008; 79:163-169.

33. The Diabetes Prevention Program (DPP) Research Group. Description of lifestyle intervention. Diabetes Care 2002 Dec.; 25(12): 2165-2171.

34. KnowlerWC, Barrett-Connor E, Fowler SE, et al. Reduction in the incidence of type 2 diabetes with lifestyle intervention or metformin. N. Engl. J. Med. 2002; 346:393-403.

35. Gordon Smith A, Russell J, Feldman EL, et al. Lifestyle intevention for pre-diabetic neuropathy. Diabetes Care 2006; 29: 6 , 1294-1299. 